\title{
Adolescents with tetralogy of Fallot: neuropsychological assessment and structural brain imaging
}

\author{
David C. Bellinger, ${ }^{1,2}$ Michael J. Rivkin, ${ }^{1,3}$ David DeMaso, ${ }^{2,4}$ Richard L. Robertson, ${ }^{3}$ Christian Stopp, ${ }^{4}$ \\ Carolyn Dunbar-Masterson, ${ }^{4}$ David Wypij, ${ }^{4,5}$ Jane W. Newburger ${ }^{4,6}$ \\ ${ }^{1}$ Department of Neurology; ${ }^{2}$ Department of Psychiatry, Boston Children's Hospital, Harvard Medical School; \\ ${ }^{3}$ Department of Radiology; ${ }^{4}$ Department of Cardiology, Boston Children's Hospital; ${ }^{5}$ Department of Biostatistics, Harvard \\ School of Public Health; ${ }^{\circ}$ Department of Pediatrics, Harvard Medical School, Boston, Massachusetts, United States of America
}

\begin{abstract}
Background: Few data are available on the neuropsychological, behavioural, or structural brain imaging outcomes in adolescents who underwent corrective surgery in infancy for tetralogy of Fallot. Methods: In this single-centre cross-sectional study, we enrolled 91 adolescents (13-16 years old) with tetralogy of Fallot and 87 referent subjects. Assessments included tests of academic achievement, memory, executive functions, visual-spatial skills, attention, and social cognition, as well as brain magnetic resonance imaging. Results: Genetic abnormalities or syndromes were present in $25 \%$ of tetralogy of Fallot patients, who had markedly greater neuropsychological morbidities than did patients without a syndrome. However, even patients without a syndrome performed significantly worse than the referent group or population norms in all of the neuropsychological domains assessed. In multivariable regression in those without a genetic/phenotypic syndrome, the strongest predictors of adverse late neurodevelopmental outcomes included a greater number of complications at the first operation, more total surgical complications across all operations, and occurrence of post-operative seizures. The presence of at least one abnormality on structural magnetic resonance imaging was more frequent in tetralogy of Fallot patients than the referent group ( $42 \%$ versus 8\%). Conclusions: Adolescents with tetralogy of Fallot are at increased neurodevelopmental risk and would benefit from ongoing surveillance and educational supports even after childhood.
\end{abstract}

Keywords: Tetralogy of Fallot; neuropsychology; magnetic resonance imaging

Received: 9 October 2013; Accepted: 19 December 2013; First published online: 11 February 2014

A DRAMATIC INCREASE IN THE NUMBER OF SURVIVORS of paediatric cardiac surgery has been accompanied by recognition of their neurodevelopmental morbidities and the associated personal and societal costs. ${ }^{1}$ The majority of patients with tetralogy of Fallot, which accounts for $8-10 \%$ of all congenital heart lesions, ${ }^{2}$ survive to adulthood, but limited data are available on their long-term neurocognitive outcomes. They manifest deficits as early as infancy, ${ }^{3,4}$ with poorer outcomes associated with a genetic/phenotypic diagnosis, pulmonary atresia, and

Correspondence to: D. C. Bellinger, Boston Children's Hospital, 300 Longwood Avenue, Boston, MA 02115, United States of America. Tel: +(617)355 6565; Fax: +(617)730-0618; E-mail: david.bellinger@childrens.harvard.edu the apolipoprotein epsilon allele. ${ }^{5}$ At 4 years of age, $20 \%$ of children with repaired tetralogy of Fallot have a significant impairment in at least one neurodevelopmental domain, ${ }^{6}$ and at school age many have difficulties with motor skills, expressive language, speech, executive control, memory, attention, and visual-motor skills. ${ }^{6-10}$ In adulthood, patients showed reduced IQ scores ${ }^{11}$ and deficits in executive functions. ${ }^{12}$ Patients with the $22 \mathrm{q} 11$ microdeletion are at substantial risk of low IQ and psychiatric disorders. ${ }^{12}$

We report here on neuropsychological and structural neuroimaging evaluations of 91 adolescents who underwent surgery in infancy for tetralogy of Fallot, comparing their outcomes to those of the general population and a local referent group. 


\section{Methods}

Subjects

We enrolled patients from 2004 to 2008 at Boston Children's Hospital. Admission criteria included age 13-16 years, diagnosis of tetralogy of Fallot with or without pulmonary atresia, and an interval of at least 3 months between the last cardiac surgery and neurodevelopmental testing. We excluded patients with disorders that would prevent completion of the study assessments - that is, metal implants trisomy 21 , or lack of reading fluency by the primary caregiver.

We recruited a group of 13-16-year-old, normally developing, referents to whom patients were compared with regard to brain magnetic resonance imaging and psychometric tests lacking national norms. The referents were not matched to the tetralogy of Fallot patients on any factors. Exclusion criteria were similar to those used in the National Institutes of Health's magnetic resonance imaging study of normal brain development. ${ }^{13}$ Referents were recruited from a variety of sources, including local paediatric practices, posted notices, and personal referral.

The study was approved by the hospital Institutional Review Board; informed consent was obtained from parents/guardians, and assent was obtained from adolescents.

\section{Neuropsychological assessment}

Intelligence. The endpoints were the five composite scores of the Wechsler Intelligence Scale for Children-Fourth Edition ${ }^{14}$ : Full-Scale IQ, Verbal Comprehension, Perceptual Reasoning, Working Memory, Processing Speed.

Academic achievement. The endpoints were the Reading Composite and Math Composite scores of the Wechsler Individual Achievement Test-Second Edition. ${ }^{15}$ Tables in the Wechsler Intelligence Scale for Children-Fourth Edition Technical and Interpretive Manual $^{16}$ were used to identify scores that differ significantly from the score predicted based on an individual's Full-Scale IQ score.

Memory. The primary endpoint was the General Memory Index of the Children's Memory Scale. ${ }^{17}$

Executive functions. A summary score was calculated by averaging scores on five subtests of the Delis-Kaplan Executive Function System ${ }^{18}$ : Verbal Fluency (mean score on the letter and semantic fluency trials), Design Fluency (primary combined measure), Sorting (combined conditions score), Word Context (consecutively correct score), and Tower (total achievement score). Adolescents, parents, and teachers completed the Behavior Rating Inventory of Executive Function. ${ }^{19-20}$ The General Executive Composite score was analysed.
Visual-Spatial skills. A Visual-Spatial summary score was calculated by averaging scores on the seven subscales of the Test of Visual-Perceptual Skills (non-motor) (Upper Level)-Revised. ${ }^{21}$ The copy, immediate recall, and delayed recall trials of the Rey-Osterrieth Complex Figure were scored for Organization, Structural Elements, and Incident Elements. $^{22}$ Adolescents completed the Sense of Directions Scale. ${ }^{23}$

Attention. The primary score was the Attention Deficit Hyperactivity Disorder Index score on a parent-completed questionnaire. ${ }^{24}$

Social cognition. On the Reading the Mind in the Eyes Test-Revised, ${ }^{25}$ an adolescent judged the emotions expressed in photographs of the eye region. The Autism Spectrum Quotient, ${ }^{26}$ a self-report questionnaire, assesses autistic traits in the general population. The Toronto Alexithymia Scale, ${ }^{27}$ a self-report questionnaire assessing emotional deficits, generates three scores: Difficulty Identifying Feelings, Difficulty Describing Feelings, and Externally Oriented Thinking.

\section{Magnetic resonance imaging}

Magnetic resonance imaging was performed on a 1.5 T General Electric Twinspeed magnetic resonance scanner at the 13.0 hardware/software configuration (General Electric Medical Systems, Milwaukee, Wisconsin, United States of America). Adolescents were imaged with 3D-volumetric and dual echo magnetic resonance imaging during the same scanning session. Following acquisition of the 3D volumetric T1-weighted high-resolution 3D Fourier Transform Spoiled Gradient data, high-resolution proton density and T2-weighted images were obtained. The spoiled gradient neuroanatomic data were obtained (field of view, $24 \mathrm{~cm}$; contiguous slice thickness, $1.5 \mathrm{~mm}$; slices, 120; repetition time/echo time, 40/4 seconds; matrix, $256 \times 192$; flip angle, 20 $)$ in 10 minutes 20 seconds. The $\mathrm{T} 2$-weighted and proton density data were acquired using a dual-echo fast-spin echo pulse sequence (echo train length, 8; 3-mm skip, 3-mm interleaved; 2 acquisitions; repetition time/echo time/ second echo time, 4000/14/84 seconds; $256 \times 192$; field of view, $20 \mathrm{~cm}$; number of excitations, 1) in 6 minutes, 25 seconds. Whole-brain susceptibility weighted imaging data were acquired in 2 minutes, 20 seconds.

Magnetic resonance imaging scans were evaluated by a neuroradiologist (R.L.R.) blinded to the participant group. Images were assessed by visual inspection to rate the quality of data and to identify abnormalities. Abnormalities were classified by origin (i.e., acquired, developmental), type (i.e., infarction, mineralization, iron deposition, myelination delay, ventriculomegaly, abnormal T2W signal hyperintensity), extent (i.e., focal or diffuse), and anatomic location. 


\section{Genetic classification}

Tetralogy of Fallot patients were assigned to the genetic/ phenotypic syndrome group based on (1) medical history of a genetic/phenotypic syndrome of multiple anomalies or (2) a finding of a genetic disorder, such as 22q11 microdeletion, on formal testing.

\section{Statistical methods}

Comparisons of operative and medical risk factors, special services, cardiac symptomatology, and magnetic resonance imaging variables were made using Fisher's exact tests for categorical measures and either twosample t-tests for continuous measures when normally distributed or Kruskal-Wallis tests when non-normal. Comparisons with respect to NYHA class utilised Cochran-Armitage trend tests. Neuropsychological outcomes of each syndrome group were compared with expected population means when available using onesample t-tests. Linear regression with adjustment for concurrent family social class - Hollingshead FourFactor Index - was used to compare neuropsychological outcomes of the syndrome groups to those of the referent group when a population mean was not available - that is Rey-Osterrieth Complex Figure, Sense of Direction Scale, Reading the Mind in the Eyes Test-Revised, Autism Spectrum Quotient - and for such comparisons between the syndrome groups.

Linear regression analyses were used to identify predictors of neuropsychological outcomes of adolescents with tetralogy of Fallot without a genetic/phenotypic syndrome, using the variables listed in Table 1 - except NYHA classification and any neurological event. Predictors were screened to identify associations with the outcomes having a $p<0.20$, adjusting for family class. Predictors meeting this criterion were included in stepwise backward analysis with a $\mathrm{p}<0.05$ retention criterion. Similar procedures were used to identify factors predicting magnetic resonance imaging outcomes using logistic regression. Surgical complications included cardiac arrest, choreoathetosis, need for extracorporeal membrane oxygenation, junctional ectopic tachycardia, necrotising enterocolitis, prolonged intensive care unit course $(>1$ week), renal failure (serum creatinine $>1.5 \mathrm{mg} / \mathrm{dL}$ ), re-operation/exploration, respiratory arrest, seizures, stroke, ventricular fibrillation, ventricular tachycardia, re-intubation, pneumonia, bleeding/haemorrhage, open chest, sepsis, ventricular premature beats, supraventricular tachycardia, "TET spells" during surgery, and tamponade.

\section{Results}

Cardiac status and bistory

Of 184 patients with tetralogy of Fallot seen at our institution and who met study eligibility criteria,
$39(21 \%)$ were followed up elsewhere or lost to follow-up, and 54 (29\%) declined study participation. Our study cohort included the remaining 91 patients, as well as 87 referent adolescents. Table 1 presents selected aspects of the medical histories of

Table 1. Characteristics of adolescents with tetralogy of Fallot $(\mathrm{n}=91)$.

\begin{tabular}{|c|c|}
\hline Variables & $\begin{array}{c}\%, \text { mean } \pm \mathrm{SD} \\
\text { or median } \\
\quad(\text { range })\end{array}$ \\
\hline \multicolumn{2}{|l|}{ Pre-operative characteristics } \\
\hline Birth weight $(\mathrm{kg})$ & $3.1 \pm 0.7$ \\
\hline Gestational age (weeks) & $39.2 \pm 2.3$ \\
\hline Male & $55^{*}$ \\
\hline Race/ethnicity (Caucasian) & $87 *$ \\
\hline Genetic diagnosis present & 25 \\
\hline Pulmonary atresia & 29 \\
\hline \multicolumn{2}{|l|}{ Status at first surgery } \\
\hline Age (days) & $112(2-1378)$ \\
\hline Age $>30$ days & 76 \\
\hline Open procedure & 88 \\
\hline Duration of DHCA (minutes), if open & $30(0-69)$ \\
\hline Total support time (minutes), if open & $97(21-220)$ \\
\hline \multicolumn{2}{|l|}{ Number of surgical complications } \\
\hline None & 53 \\
\hline One & 26 \\
\hline Two or more & 21 \\
\hline \multicolumn{2}{|l|}{ Medical history } \\
\hline \multicolumn{2}{|l|}{ Total number of surgeries } \\
\hline One & 48 \\
\hline Two & 24 \\
\hline Three or more & 27 \\
\hline \multicolumn{2}{|l|}{ Total number of open surgeries } \\
\hline One & 54 \\
\hline Two & 30 \\
\hline Three or more & 16 \\
\hline \multicolumn{2}{|l|}{ Total number of surgical complications } \\
\hline None & 48 \\
\hline One & 21 \\
\hline Two or more & 31 \\
\hline \multicolumn{2}{|l|}{ Total number of catheterisations } \\
\hline None & 26 \\
\hline One or two & 30 \\
\hline Three or more & 44 \\
\hline \multicolumn{2}{|c|}{ Total number of catheterisation complications } \\
\hline None & 68 \\
\hline One or two & 13 \\
\hline Three or more & 19 \\
\hline Seizures & 12 \\
\hline Any neurological event** & 20 \\
\hline \multicolumn{2}{|l|}{ New York Heart Association class } \\
\hline Class I & 68 \\
\hline Class II & 29 \\
\hline Class III & 3 \\
\hline Family social status $* * *$ & $49 \pm 12 *$ \\
\hline Age at developmental testing (years) & $14.6 \pm 1.2 *$ \\
\hline \multicolumn{2}{|c|}{$\begin{array}{l}* \text { Referent group: male, } 50 \% \text {; Caucasian, } 81 \% \text {; family social class, } \\
53 \pm 10 \text {, age at testing, } 15.3 \pm 1.1 \text { years } \\
* * \text { Includes stroke, seizure, choreoathetosis, and meningitis } \\
* * * \text { Score on Hollingshead Four Factor Index of Social Status, with } \\
\text { higher scores indicating higher social status }\end{array}$} \\
\hline
\end{tabular}


the patients. Most (76\%) were older than 30 days at their first operation, slightly fewer than half experienced $\geqslant 1$ complications during the first surgery, and more than half had undergone $\geqslant 2$ surgeries (median number 2 , range $1-7)$. Three-quarters $(74 \%)$ had undergone $\geqslant 1$ cardiac catheterisation (median 2, range $0-14$ ), and $32 \%$ experienced $\geqslant 1$ complications during a catheterisation. One-quarter $(25 \%)$ had a genetic/phenotypic syndrome, including 22q11 $(\mathrm{n}=11)$, VATER/VACTERL $(\mathrm{n}=8)$, foetal alcohol syndrome $(n=2)$, Alagille's syndrome $(n=1)$, and Turner's syndrome $(n=1)$. In all, $26(29 \%)$ patients had pulmonary atresia. The syndrome and nonsyndrome groups were comparable in operative measures and other medical risk factors. Those with pulmonary atresia, compared with those with pulmonary stenosis, were more likely to be neonates at the first operation $(\mathrm{p}<0.001)$ and to have had a first operation that was closed, that is, performed off bypass $(\mathrm{p}=0.01)$. They also had more total operations and catheterisations ( $\mathrm{p}<0.001$ each), as well as more surgical and catheterisation complications $(\mathrm{p}=0.03$ and $\mathrm{p}<0.001$, respectively). They were not more likely than patients without pulmonary atresia to be in the genetic/phenotypic syndrome group $(\mathrm{p}=0.80)$.

Among patients, medical history was significant for stroke $(n=4)$, seizures $(n=11)$, choreoathetosis $(\mathrm{n}=4)$, and meningitis $(\mathrm{n}=2)$. Parents reported that $19 \%(\mathrm{n}=17)$ had been diagnosed with attention deficit hyperactivity disorder $(16 \%$ of those without a genetic/phenotypic syndrome, $26 \%$ of those with a syndrome) and $35 \%(\mathrm{n}=32)$ with a learning disability, which was more common in those with a syndrome $(13 / 23,56 \%)$ than those without a syndrome $(19 / 68,28 \% ; \mathrm{p}=0.02)$.

Compared with referent subjects, adolescents with tetralogy of Fallot were more likely to have received any special services $(82 \%$ versus $49 \%, \mathrm{p}<0.001)$, including tutoring $(52 \%$ versus $26 \%, \mathrm{p}<0.001)$, early intervention ( $40 \%$ versus $1 \%, \mathrm{p}<0.001)$, occupational therapy ( $44 \%$ versus $0 \%, \mathrm{p}<0.001$ ), special education ( $42 \%$ versus $1 \%, \mathrm{p}<0.001)$, psychotherapy or counselling $(36 \%$ versus $15 \%$, $\mathrm{p}=0.002)$, and physical therapy (35\% versus $19 \%$, $\mathrm{p}=0.02)$. Approximately one in four (24\%) patients, compared with $2 \%$ of referents, had been retained in grade $(\mathrm{p}<0.001)$. Receipt of neurodevelopmental services was more frequent in the group with a syndrome than in the group without a syndrome: early intervention (70\% versus $29 \%$, $\mathrm{p}=0.001)$, occupational therapy $(65 \%$ versus $37 \%$, $\mathrm{p}=0.03)$, and physical therapy $(61 \%$ versus $26 \%$, $\mathrm{p}=0.005)$. Reports of special services were comparable between patients with and without pulmonary atresia.
Most adolescents with tetralogy of Fallot were in NYHA class I (68\%), whereas $29 \%$ were in class II, and $3 \%$ in class III, whereas nearly all referent subjects were in class I (97\%), with the remaining 3\% in class II $(\mathrm{p}<0.001)$. NYHA class was not associated with the presence of a genetic/phenotypic syndrome, but was significantly worse for the group with pulmonary atresia compared with those with pulmonary stenosis $(\mathrm{p}=0.01)$. With regard to cardiac symptoms, patients were more likely than referents to report exercise intolerance $(24 \%$ versus $2 \%, \mathrm{p}<0.001)$, shortness of breath with exercise $(32 \%$ versus $16 \%$, $\mathrm{p}=0.02)$, palpitations $(20 \%$ versus $1 \%, \mathrm{p}<0.001)$, chest pain $(22 \%$ versus $3 \%, \mathrm{p}<0.001)$, and general fatigue $(24 \%$ versus $1 \%, \mathrm{p}<0.001)$. Reports of cardiac symptoms were similar between syndrome groups. Patients with pulmonary atresia were more likely than patients without pulmonary atresia to report shortness of breath with exercise ( $54 \%$ versus $23 \%, \mathrm{p}=0.006$ ). There were three patients (3\%) who had a pacemaker. Medications included angiotensin-converting enzyme inhibitors ( $\mathrm{n}=4), \beta$-blockers $(\mathrm{n}=5)$, digoxin $(\mathrm{n}=8)$, diuretics $(n=4)$, medication for attention deficit hyperactivity disorder $(\mathrm{n}=10)$, and psychotropic medications $(n=5)$. There were two adolescents with tetralogy of Fallot who reported use of both attention deficit hyperactivity disorder and psychotropic medications.

\section{Neuropsychological assessment}

The mean age (standard deviation) of the patients was 14.6 (1.2) years. Table 2 summarises the test scores of all patients and scores stratified by the presence of a genetic/phenotypic syndrome.

\section{General intelligence}

Scores of patients without a genetic/phenotype diagnosis were lower than the expected population means for each index from the Wechsler Intelligence Scale for Children-Fourth Edition except Verbal Comprehension, with differences of $6-10$ points $(0.4-0.7$ standard deviation). Almost a third of patients (31\%) had a FullScale IQ score of $\leqslant 85$ (compared with an expected frequency of $16 \%$ ) and $16 \%$ had a score $\leqslant 70$ (compared with an expected $2 \%$ ).

Scores of adolescents with a syndrome were significantly lower than those of adolescents without a syndrome on all composites, with differences approaching, and in some cases exceeding, one standard deviation. Half of these adolescents had a Full-Scale IQ score $\leqslant 85$, and $36 \%$ a score $\leqslant 70$.

\section{Academic achievement}

The Reading and Math Composite scores of adolescents without a genetic/phenotypic diagnosis were 


\begin{tabular}{|c|c|c|c|c|c|c|}
\hline \multirow[b]{2}{*}{ Variables } & \multicolumn{3}{|c|}{ Tetralogy of Fallot } & \multirow[b]{2}{*}{$\begin{array}{l}\text { p-value comparing } \\
\text { those with genetic } \\
\text { diagnosis to those without* }\end{array}$} & \multirow[b]{2}{*}{$\begin{array}{l}\text { p-value comparing those } \\
\text { without genetic diagnosis } \\
\text { to expected population mean** }\end{array}$} & \multirow[b]{2}{*}{$\begin{array}{l}\text { p-value comparing those with } \\
\text { genetic diagnosis to expected } \\
\text { population mean** }\end{array}$} \\
\hline & All $(\mathrm{n}=91)$ & $\begin{array}{l}\text { Genetic diagnosis } \\
\text { absent or no } \\
\text { evaluation }(n=68)\end{array}$ & $\begin{array}{l}\text { Genetic diagnosis } \\
\text { present }(\mathrm{n}=23)\end{array}$ & & & \\
\hline & & Mean \pm SD & & & & \\
\hline \multicolumn{7}{|l|}{ Wechsler Intelligence Scale } \\
\hline Verbal Comprehension & $95.6 \pm 21.5$ & $98.7 \pm 20.4$ & $86.4 \pm 22.5$ & 0.01 & 0.62 & 0.009 \\
\hline Perceptual Reasoning & $89.1 \pm 21.2$ & $93.7 \pm 20.2$ & $75.4 \pm 18.2$ & $<0.001$ & 0.01 & $<0.001$ \\
\hline Working Memory & $88.9 \pm 21.0$ & $91.4 \pm 20.2$ & $81.7 \pm 22.1$ & 0.046 & $<0.001$ & $<0.001$ \\
\hline Processing Speed & $87.0 \pm 19.3$ & $90.0 \pm 18.4$ & $77.8 \pm 19.5$ & 0.008 & $<0.001$ & $<0.001$ \\
\hline Full-Scale IQ & $89.0 \pm 22.8$ & $93.0 \pm 21.4$ & $77.0 \pm 23.1$ & 0.002 & 0.009 & $<0.001$ \\
\hline \multicolumn{7}{|l|}{ Wechsler Individual Achievement Test } \\
\hline Reading Composite & $92.6 \pm 20.3$ & $96.1 \pm 17.8$ & $82.5 \pm 24.1$ & 0.003 & 0.07 & 0.002 \\
\hline Mathematics Composite & $89.5 \pm 27.6$ & $95.1 \pm 25.6$ & $73.1 \pm 27.1$ & $<0.001$ & 0.12 & $<0.001$ \\
\hline General Memory Index & $86.3 \pm 21.7$ & $88.5 \pm 20.5$ & $79.3 \pm 24.3$ & 0.09 & $<0.001$ & $<0.001$ \\
\hline Executive Function Summary Score & $8.2 \pm 2.9$ & $8.7 \pm 2.8$ & $6.4 \pm 2.7$ & $<0.001$ & $<0.001$ & $<0.001$ \\
\hline \multicolumn{7}{|c|}{ Behavior Rating Inventory of Executive Function } \\
\hline Self-report & $50.3 \pm 10.8$ & $49.8 \pm 10.8$ & $51.9 \pm 10.9$ & 0.43 & 0.89 & 0.45 \\
\hline Parent & $58.0 \pm 13.1$ & $55.8 \pm 12.8$ & $64.4 \pm 12.1$ & 0.004 & $<0.001$ & $<0.001$ \\
\hline Teacher & $63.0 \pm 19.7$ & $62.3 \pm 19.8$ & $65.4 \pm 20.1$ & 0.49 & $<0.001$ & 0.01 \\
\hline Visual-Spatial Summary Score & $82.6 \pm 19.7$ & $86.4 \pm 19.6$ & $70.9 \pm 15.2$ & $<0.001$ & $<0.001$ & $<0.001$ \\
\hline \multicolumn{7}{|l|}{$\begin{array}{l}\text { visual-spatial Summary score } \\
\text { Rey-Osterrieth Complex Figure*** }\end{array}$} \\
\hline Copy: Organisation & $7.8 \pm 3.8$ & $8.7 \pm 3.7$ & $5.1 \pm 3.0$ & $<0.001$ & 0.001 & $<0.001$ \\
\hline Structural Element & $23.2 \pm 3.8$ & $23.7 \pm 3.2$ & $21.6 \pm 4.9$ & 0.02 & 0.004 & $<0.001$ \\
\hline Incidental Element & $36.3 \pm 6.0$ & $37.3 \pm 4.0$ & $33.2 \pm 9.3$ & 0.006 & 0.003 & $<0.001$ \\
\hline Immediate Recall: Organisation & $6.2 \pm 4.1$ & $6.8 \pm 4.1$ & $3.9 \pm 3.5$ & 0.006 & 0.008 & $<0.001$ \\
\hline Structural Element & $19.8 \pm 6.0$ & $21.0 \pm 4.8$ & $15.7 \pm 7.8$ & $<0.001$ & 0.03 & $<0.001$ \\
\hline Incidental Element & $26.4 \pm 9.1$ & $27.7 \pm 8.1$ & $21.8 \pm 11.0$ & 0.01 & 0.46 & $<0.001$ \\
\hline Delayed Recall: Organisation & $6.4 \pm 4.0$ & $7.0 \pm 3.8$ & $4.2 \pm 3.7$ & 0.005 & 0.048 & $<0.001$ \\
\hline Structural Element & $19.6 \pm 6.5$ & $21.0 \pm 5.3$ & $15.2 \pm 8.0$ & $<0.001$ & 0.004 & $<0.001$ \\
\hline Incidental Element & $25.5 \pm 9.2$ & $27.1 \pm 8.1$ & $20.1 \pm 10.8$ & 0.003 & 0.30 & $<0.001$ \\
\hline Sense of Direction Scale*** & $46.2 \pm 9.0$ & $46.1 \pm 8.5$ & $46.3 \pm 10.8$ & 0.97 & 0.09 & 0.29 \\
\hline Parent Conner's ADHD Index T Score & $59.3 \pm 13.6$ & $57.2 \pm 12.8$ & $65.5 \pm 14.3$ & 0.007 & $<0.001$ & $<0.001$ \\
\hline Reading the Mind in the Eyes*** & $18.8 \pm 5.9$ & $19.2 \pm 6.0$ & $17.0 \pm 5.0$ & 0.38 & $<0.001$ & $<0.001$ \\
\hline \multicolumn{7}{|l|}{ Toronto Alexithymia Scale**** } \\
\hline Difficulty Identifying Feelings & $17.3 \pm 6.0$ & $16.7 \pm 6.0$ & $19.2 \pm 6.1$ & 0.42 & 0.049 & 0.01 \\
\hline Difficulty Describing Feelings & $13.7 \pm 4.8$ & $12.9 \pm 4.3$ & $16.8 \pm 5.5$ & 0.09 & 0.88 & 0.04 \\
\hline Externally Oriented Thinking & $20.6 \pm 4.7$ & $20.2 \pm 4.3$ & $22.0 \pm 5.8$ & 0.40 & 0.93 & 0.41 \\
\hline Autism Spectrum Quotient**** & $17.9 \pm 5.6$ & $17.6 \pm 5.9$ & $18.9 \pm 4.2$ & 0.31 & 0.03 & 0.01 \\
\hline
\end{tabular}

* Determined by linear regression with adjustment for concurrent family social status

**Determined by one-sample t-tests comparing the tetralogy of Fallot group - with or without genetic diagnosis - with expected population means of 100, 10, or 50, as appropriate

***Expected population means are not available; p-value is determined by linear regression comparing the tetralogy of Fallot group - with or without genetic diagnosis - with a group of 87 referents, with adjustment for concurrent family social status. The scores (mean \pm standard deviation) of the referents were as follows:

Rey-Osterrieth Complex Figure

Copy: Organisation $10.8 \pm 2.6$, Structural Element: $24.9 \pm 0.5$, Incidental Element: $38.8 \pm 0.6$

Immediate Recall: Organisation: $8.9 \pm 3.8$, Structural Element: $23.4 \pm 3.2$, Incidental Element: $28.9 \pm 6.6$

Delayed Recall: Organisation: $8.7 \pm 3.5$, Structural Element: $23.8 \pm 3.5$, Incidental Element: $29.0 \pm 6.5$

Sense of Direction Scale: $50.2 \pm 10.2$

Read the Mind in the Eyes Test-Revised: $23.5 \pm 4.7$

Autism Spectrum Quotient: $14.9 \pm 5.9$

Missing $<10 \%$ of outcomes except for Behavior Rating Inventory of Executive Function - Teacher $(n=58)$, Reading the Mind in the Eyes $(n=66)$ and the Toronto Alexithymia Scale $(n=27)$ tasks. 
slightly lower, though not significantly, than the expected population means. On the Reading Composite, $21 \%$ scored $\leqslant 85$ and $6 \%$ scored $\leqslant 70$. On the Math Composite, $29 \%$ scored $\leqslant 85$ and $19 \%$ scored $\leqslant 70$. The frequencies of scores significantly lower than expected based on Full-Scale IQ score were 23\% for Reading Composite and 29\% for Math Composite.

Reading Composite and Mathematics Composite scores of adolescents with a syndrome were significantly lower than scores of adolescents without a syndrome, with the deficit being substantially greater on mathematics than reading. Compared with the expected population, the average deficit on the Mathematics Composite score for those with a syndrome was 27 points (1.8 standard deviation). Half (52\%) scored $\leqslant 85$ on the Reading Composite and $26 \%$ scored $\leqslant 70$. The corresponding figures for the Math Composite were $57 \%$ and $48 \%$, respectively. On the Reading Composite, $18 \%$ scored significantly lower than would be expected based on Full-Scale IQ, and 55\% scored significantly lower on the Math Composite.

\section{Memory}

The mean General Memory Index score of adolescents without a genetic/phenotypic diagnosis was $\sim 0.8$ standard deviation lower than the expected population mean. Almost half (47\%) scored $\leqslant 85$ and $20 \%$ scored $\leqslant 70$. In general, verbal memory scores exceeded visual memory scores, for both immediate and delayed trials.

The scores of adolescents with a genetic/phenotypic syndrome tended to be lower than those without a syndrome, with a mean difference of approximately two-thirds of a standard deviation. The mean score was $\sim 1.4$ standard deviations lower than the expected population mean; $57 \%$ of adolescents scored $\leqslant 85$ and $38 \%$ scored $\leqslant 70$.

\section{Executive Functions}

The Executive Function Summary scores of adolescents without a genetic/phenotypic syndrome were significantly lower than the expected population mean of $10 ; 22 \%$ scored $\geqslant 1$ standard deviation (i.e., 3 points) below the expected mean, and $7 \%$ scored $\geqslant 2$ standard deviations or below the expected mean. The mean score of adolescents with a syndrome was significantly lower than that of adolescents without a syndrome; $48 \%$ scored $\geqslant 1$ standard deviation below the expected mean, and $29 \%$ scored $\geqslant 2$ standard deviations or more below.

On the Behavior Rating Inventory of Executive Function, both parent- and teacher-reported General Executive Composite scores were higher - that is, more problematic - than the expected population mean. For adolescents without a genetic/phenotypic syndrome, teachers tended to report more problems than parents. On the parent questionnaire, $27 \%$ of adolescents without a genetic/phenotypic syndrome had a score $\geqslant 65$ - "of potential clinical significance" compared with $48 \%$ of adolescents with a syndrome. On the teacher questionnaire, these figures were $36 \%$ and $50 \%$, respectively. On the adolescent self-report questionnaire, those without and with a syndrome scored similarly $-12 \%$ and $11 \%$ of scores, respectively, were in the range of potential clinical significance. Remarkably, neither group differed significantly from the expected population mean.

\section{Visual-spatial skills}

Patients scored significantly lower than the expected population mean on the Visual-Spatial Summary score, with the difference corresponding to 1 standard deviation for adolescents without a genetic/phenotypic diagnosis and 2 standard deviation for adolescents with a genetic/phenotypic syndrome. On the Rey-Osterrieth Complex Figure, adolescents with a syndrome scored significantly lower than those without a syndrome on all three endpoints for each condition - that is, Copy, Immediate Recall, Delayed Recall.

On the Sense of Direction Scale, scores of adolescents in the two syndrome groups did not differ significantly, nor did the scores of either group differ significantly from those of the referent group.

\section{Attention}

Attention Deficit Hyperactivity Disorder Index scores of the adolescents without a genetic/phenotypic syndrome were $\sim 0.75$ standard deviation higher - that is less optimal - than the expected population mean. Approximately one in five $(21 \%)$ scored $\geqslant 66$, which is considered to be "moderately atypical". ${ }^{24}$

Attention Deficit Hyperactivity Disorder Index scores of the adolescents with a syndrome were significantly higher than those of adolescents without a syndrome, with the difference in means being 0.8 standard deviation, and $\sim 1.5$ standard deviation higher than the expected population mean. Almost half $(48 \%)$ scored $\geqslant 66$.

\section{Social cognition}

On the Reading the Mind in the Eyes Test-Revised, scores of the syndrome groups did not differ significantly, but both groups scored significantly lower (worse) than the referent group. This pattern was also evident for scores on the Autism Spectrum Quotient, with adolescents with tetralogy of Fallot scoring significantly higher (worse) than referents. Scores on the Toronto Alexithymia Scale were similar in the two syndrome groups, but the adolescents with a syndrome scored significantly higher (worse) than 
Table 3. Structural magnetic resonance imaging findings of adolescents with tetralogy of Fallot and referents.

\begin{tabular}{|c|c|c|c|}
\hline Variables & TOF $(n=66)$ & Referent $(\mathrm{n}=75)$ & p-value* \\
\hline \multicolumn{4}{|c|}{$\mathrm{n}(\%)$} \\
\hline Any abnormality & $28(42)$ & $6(8)$ & $<0.001$ \\
\hline Focal or multifocal abnormality & $24(36)$ & $1(1)$ & $<0.001$ \\
\hline Focal infarction or atrophy & $4(6)$ & 0 & 0.046 \\
\hline Brain mineralisation/iron deposit & $21(32)$ & $1(1)$ & $<0.001$ \\
\hline Diffuse abnormality & $2(3)$ & $1(1)$ & 0.60 \\
\hline Myelination delay & 0 & 0 & - \\
\hline Ventriculogealy & $1(2)$ & 0 & 0.47 \\
\hline Abnormal T2 hyperintensities & $1(2)$ & $1(1)$ & 1.0 \\
\hline Generalised abnormality & $1(2)$ & 0 & 0.47 \\
\hline Developmental abnormality & $6(9)$ & $4(5)$ & 0.52 \\
\hline Major malformation** & $1(2)$ & 0 & 0.47 \\
\hline Minor malformation $* * *$ & $5(8)$ & $4(5)$ & 0.73 \\
\hline
\end{tabular}

TOF $=$ tetralogy of Fallot

*Determined by Fisher's exact tests

**The major malformation is hypoplasia in the right cerebellar hemisphere and Dandy-Walker spectrum anomaly

***Minor malformations include agenesis of the corpus and partial anterior absence of corpus callosum - no other findings of holoprosencephaly - arachnoid cyst, subependymal nodular heteropia (right parietal white matter), absent splenium of corpus callosum (not agenesis), and mildly dysmorphic ventricles in TOF group; and Chiari malformation and developmental venous anomaly in right parietal lobe in referent group

referents on the Difficulty Identifying Feelings and Difficulty Describing Feelings subscales $(\mathrm{p}=0.01$, 0.04 , respectively).

\section{Comparison of tetralogy of Fallot subgroups with and without pulmonary atresia}

Patients with pulmonary atresia consistently performed worse than those without pulmonary atresia, with many of the relative deficits approaching, and in some cases exceeding, 0.5 standard deviation in magnitude (data not shown). For most endpoints, however, the difference was not statistically significant, presumably because of the small size of the pulmonary atresia subgroup.

\section{Magnetic resonance imaging}

Complete data were available for 66 adolescents with tetralogy of Fallot and 75 referents. The frequency of "any abnormality" was fivefold greater among the patients than the referents $(42 \%$ versus $8 \%$, $\mathrm{p}<0.001$; Table 3). The great majority of abnormalities were focal or multifocal, with most involving brain mineralisation or iron deposits. Developmental abnormalities were almost twice as common among patients as referents (9\% versus 5\%, respectively), but the difference did not reach statistical significance, with limited statistical power.

The patients with and without a genetic/phenotypic syndrome did not differ in the frequency of abnormalities. The frequency of any abnormality was greater $(\mathrm{p}<0.001)$ among patients with pulmonary atresia $(13 / 16,81 \%)$ than those without pulmonary atresia $(15 / 50,30 \%)$.

\section{Predictors of neuropsychological and magnetic resonance imaging outcomes}

Table 4 lists potential predictors that were retained in stepwise linear regression analyses of selected neuropsychological outcomes of adolescents without a genetic/phenotypic syndrome. The number of complications at first operation was associated with the greatest number of scores. For many scores, a "dose-related" increase in the deficit was observed. Compared with adolescents with no complications, those with $\geqslant 2$ complications scored an average of 17 points lower in Verbal Comprehension, and adolescents with one complication averaged 7 points lower. Similarly, adolescents with $\geqslant 2$ complications scored 29 points lower on the Mathematics Composite and 25 points lower on the Visual-Spatial Summary score, whereas those with one complication scored 11 and 13 points lower, respectively. Other covariates associated with multiple endpoints were the total number of surgical complications, gestational age, and seizures.

In analyses of three magnetic resonance imaging endpoints - that is, any abnormality, focal/multifocal abnormality, brain mineralisation/iron deposit patients with pulmonary atresia had greater odds of any abnormality (odds ratio $=10.1,95 \%$ confidence interval: $2.5-40.7)$ or a brain mineralisation deposit (odds ratio $=4.1,95 \%$ confidence interval: 1.2-13.3) (Table 5). Reduced odds of focal/multifocal abnormality were significantly associated with higher birth weight (odds ratio $=0.11,95 \%$ confidence interval: $0.01-0.87)$. The confidence intervals for all of these odds ratios are wide because of small cell counts, however. 
Table 4. Stepwise linear regression of select neuropsychological outcomes of adolescents with tetralogy of Fallot without genetic anomalies $(\mathrm{n}=68)$, adjusting for concurrent family social status.

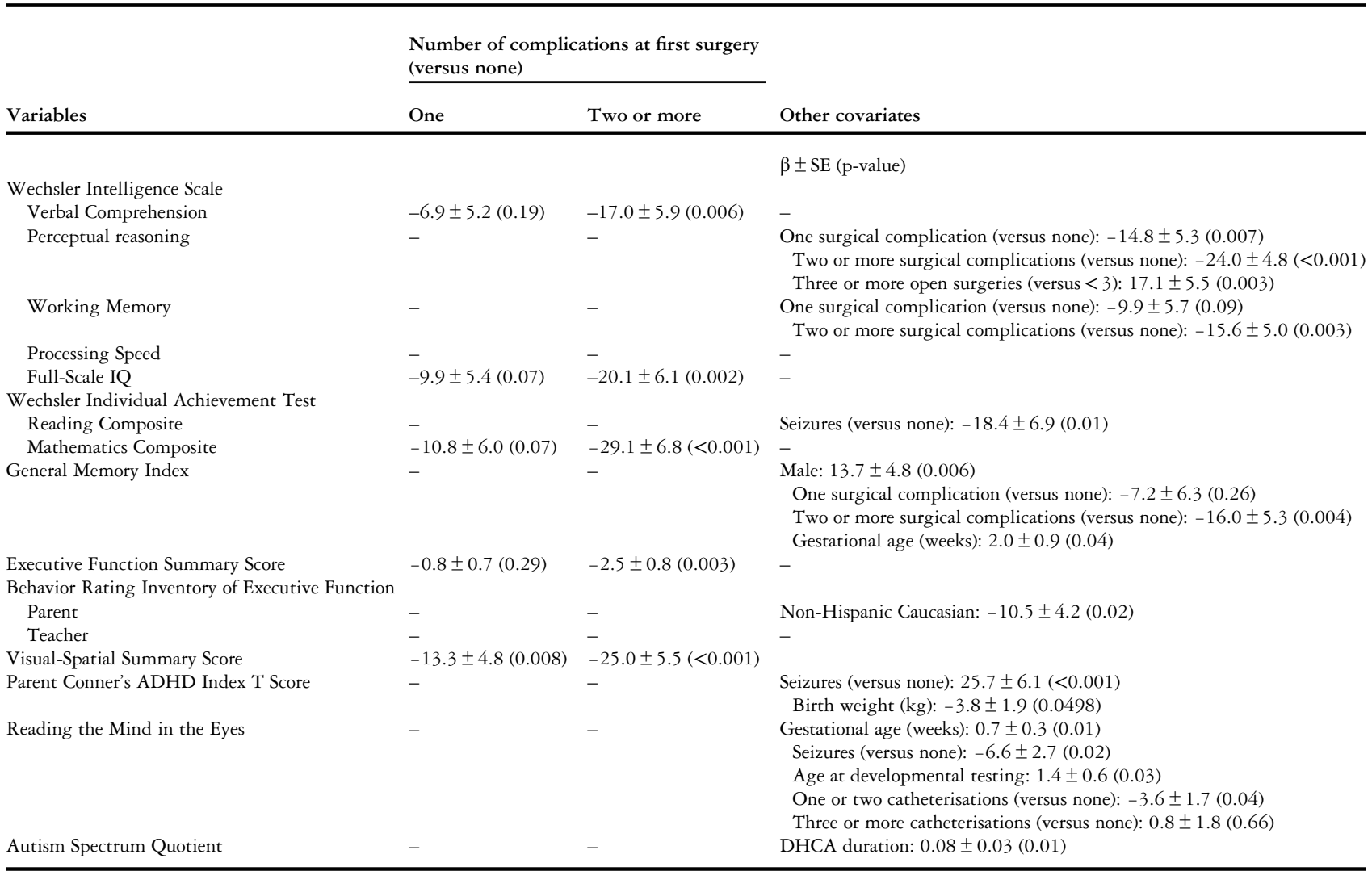

ADHD = Attention Deficit Hyperactivity Disorder; DHCA = deep hypothermic circulatory arrest

All characteristics from Table 1 were considered for inclusion in the regression models except NYHA classification and any neurological event

Coefficients for intercepts and family social status are not reported

Table 5. Stepwise logistic regression of select magnetic resonance imaging outcomes of adolescents with tetralogy of Fallot $(\mathrm{n}=66)$.

\begin{tabular}{lcc}
\hline Variable & Have pulmonary atresia (versus do not have) & Birth weight (kg) \\
\hline & $10.1(2.5,40.7), 0.001$ & Odds ratio (95\% CI), p-value \\
Any abnormality & $4.1(1.2,13.3), 0.02$ & - \\
$\begin{array}{l}\text { Focal or multifocal abnormality } \\
\text { Brain mineralisation deposit }\end{array}$ & - & - \\
\hline
\end{tabular}

Values are estimated odds ratios, $95 \%$ confidence intervals (CI), and p-values

All characteristics from Table 1 were considered for inclusion in the regression models except NYHA classification and any neurological event

\section{Discussion}

We found that a substantial proportion of adolescents with tetralogy of Fallot were experiencing difficulty meeting neurodevelopmental expectations, with many receiving remedial services, having been retained in grade, and manifesting deficits, compared with normative populations. Areas of diminished performance included general intelligence, academic achievement, memory, executive functions, visual-spatial skills, attention, and social cognition. These findings suggest that the deficits observed in patients at younger ages ${ }^{3-9}$ persist into adolescence. Because adolescent neurocognitive and behavioural function strongly predicts adult functioning, our data suggest the need to develop strategies to optimise outcomes of the growing population of adults with repaired tetralogy of Fallot.

Among patients without known genetic/phenotypic diagnoses, medical factors that had the strongest associations with neurodevelopmental outcomes included a greater number of complications at the first surgery, more total surgical complications across all operations, and occurrence of post-operative seizures. The number of complications at the first operation was 
"dose related" with many endpoints, as adolescents with two or more complications scored almost 2 standard deviations lower on some tests than patients with no complications. Adolescents who experienced post-operative seizures were more likely to be rated as having difficulties with executive functions and attention. For magnetic resonance imaging outcomes, the diagnosis of pulmonary atresia was associated with a greater likelihood of at least one structural abnormality.

In most respects, patients with a genetic/phenotypic diagnosis performed significantly worse than patients without such a diagnosis, confirming the findings of Zeltser et $\mathrm{al}^{4}$ and others, though because of small numbers we were not able to discern diagnosis-specific associations. In addition to the well-known relationship of tetralogy of Fallot to velo-cardio-facial syndrome, most often related to $22 \mathrm{q} 11$ microdeletion, tetralogy of Fallot is also associated with gene mutation or deletion - for example, NKX2.5, JAG1, TBX5, TBX1, and FOXC2. Indeed, molecular genetic analyses have defined a genetic aetiology in $>20 \%$ of patients - single gene defects and/or cytogenetic abnormalities - and unidentified monogenic mutations may account for an even greater proportion of cases. We hypothesise that as-yet unknown genetic factors involved in the aetiology of tetralogy of Fallot may also impact brain development and could explain, in part, the observation that even patients without known syndromes exhibited significant deficits.

With regard to executive functions, adolescents rated their skills as being average, but both parents and teachers identified many weaknesses, and the frequencies of scores of clinical concern were several-fold greater for these informants than for the adolescents themselves. Similar findings have been reported in adolescents with d-transposition of the great arteries, ${ }^{28}$ suggesting that relying solely on self-reports of patients with congenital heart disease might underestimate the severity of their challenges, at least in the domain of executive functions.

Our study should be interpreted in the light of its limitations. The most important is potential selection bias. Outcomes of patients whose families agreed to participate might not be representative of outcomes among all adolescents with tetralogy of Fallot. In the absence of data on outcomes of non-participants, however, the direction of a bias introduced by selective enrollment is uncertain. Genetic testing was performed in most, but not in every patient. The patients' surgeries occurred more than a decade ago, and more recent cohorts of patients with tetralogy of Fallot might have benefited from recent improvements in surgical and medical management. Although we found that a greater number of post-operative complications was associated with greater neurodevelopmental impairment, concordant with findings in other studies, ${ }^{29}$ the complications of catheterisation and surgery may have been collinear and are variable in severity.

In summary, although many adolescents with tetralogy of Fallot have neurodevelopmental outcomes within the average range, a sizeable minority do not, even among patients without a known genetic/phenotypic diagnosis. Our results support recent AHA/AAP guidelines, recommending enhanced neurodevelopmental surveillance of a child with congenital heart disease $^{30}$ so that appropriate, timely interventions can be tailored to assist the patient in achieving his or her fullest potential. Furthermore, our results suggest that such surveillance should extend beyond the age of 11-12 years, the oldest age for follow-up suggested in these guidelines.

\section{Acknowledgements}

The authors thank Drs Lisa Farley, Gloria Chiang, and Lou Baptista-Nadeau, as well as Jonathan Hirshberg, Carrie Morris, and Sneha Rao, for assistance in data collection; Kayla Monzak, Jill Cotter, Kate Henry, Naomi Lawrence-Reid, and Elizabeth Coogan, who served as research assistants; Donna Duva and Donna Donati who assisted with scheduling and other study logistics; and Kathy Alexander who provided administrative support. They also thank the families for their time and interest in participating.

\section{Financial Support}

This research was funded by the National Heart, Lung, and Blood Institute (J.W.N., P50 HL74734) and by the Farb Family Fund.

\section{Conflicts of Interest}

None.

\section{References}

1. Majnemer A, Limperopoulos C. Developmental progress of children with congenital heart defects requiring open heart surgery. Sem Pediatr Neurol 1999; 6: 12-19.

2. Loffredo CA. Epidemiology of cardiovascular malformations: prevalence and risk factors. Am J Med Genet 2000; 97: 319-325.

3. Bellinger DC, Wypij D, duPlessis AJ, et al. Developmental and neurologic effects of alpha-stat versus $\mathrm{pH}$-stat strategies for deep hypothermic circulatory arrest in infants. J Thorac Cardiovasc Surg 2001; 121: 374-383.

4. Zeltser I, Jarvik GP, Bernbaum J, et al. Genetic/phenotypic factors are important determinants of neurodevelopmental outcome after repair of tetralogy of Fallot. J Thorac Cardiovasc Surg 2008; 135: 91-97.

5. Gaynor JW, Gerdes M, Nord AS, et al. Is cardiac diagnosis a predictor of neurodevelopmental outcome after cardiac surgery in infancy? J Thorac Cardiovasc Surg 2010; 140: 1230-1237.

6. Hovels-Gurich HH, Konrad K, Skorzenski D, et al. Long-term neurodevelopmental outcome and exercise capacity after corrective surgery for tetralogy of Fallot or ventricular septal defect in infancy. Ann Thorac Surg 2006; 81: 958-966. 
7. Hovels-Gurich HH, Konrad K, Skorzenski D, Herpertz-Dahlmann B, Messmer BJ, Seghaye MC. Attentional dysfunction in children after corrective cardiac surgery in infancy. Ann Thorac Surg 2007; 83: $1425-1430$.

8. Hovels-Gurich HH, Bauer SB, Schnitker R, et al. Long-term outcome of speech and language in children after corrective surgery for cyanotic or acyanotic cardiac defects in infancy. Eur J Paediatr Neurol 2008; 12: 378-386.

9. Miatton M, De Wolf D, François K, Thiery E, Vingerhoets G. Intellectual, neuropsychological, and behavioral functioning in children with tetralogy of Fallot. J Thorac Cardiovasc Surg 2007; 133: 449-455.

10. Shampaine EL, Nadelman L, Rosenthal A, Behrendt D, Sloan H. Longitudinal psychological assessment in tetralogy of Fallot. Pediatr Cardiol 1990; 10: 135-150.

11. Daliento I, Mapelli D, Russo G, et al. Health related quality of life in adults with repaired tetralogy of Fallot: psychosocial and cognitive outcomes. Heart 2005; 91: 213-218.

12. Baker K, Vorstman JA. Is there a core neuropsychiatric phenotype in 22q11.2 deletion syndrome? Current Opinion in Neurology 2012; 25: 131-137.

13. Evans AC, Brain Development Cooperative Group. The NIH MRI study of normal brain development. NeuroImage 2006; 30: 184-202.

14. Wechsler D. WISC-IV. Administration and Scoring Manual. The Psychological Corporation, San Antonio, 2003.

15. The Psychological Corporation. Wechsler Individual Achievement Test-Second Edition. The Psychological Corporation, San Antonio, 2002.

16. Wechsler D. WISC-IV Integrated Technical and Interpretive Manual. Harcourt Assessment Inc., San Antonio, 2004.

17. Cohen MJ. Children's Memory Scale. The Psychological Corporation, San Antonio, 1997.

18. Delis DC, Kaplan E, Kramer JH. Delis-Kaplan Executive Function System. The Psychological Corporation, San Antonio, 2001.

19. Gioia GA, Isquith PK, Guy SC, Kenworthy L. The Behavior Rating Inventory of Executive Function. Psychological Assessment Resources, Odessa, FL, 2000.
20. Guy SC, Isquith PK, Gioia GA. Behavior Rating Inventory of Executive Function-Self-Report Version. Psychological Assessment Resources Inc, Lutz, FL, 2004.

21. Gardner MF. Test of Visual-Perceptual Skills (Non-Motor) (Upper Level) Revised. Hydeville. Psychological and Educational Publications Inc, CA, 1997.

22. Bernstein JH, Waber DP. Developmental scoring system for the Rey-Osterrieth Complex Figure: Professional manual. Psychological Assessment Resources Inc, Lutz, FL, 1996.

23. Hegarty M, Richardson AE, Montello DR, Lovelace K, Subbiah I. Development of a self-report measure of environmental spatial ability. Intelligence 2002; 30: 425-447.

24. Conners CK. Conners' Rating Scales-Revised. Multi-Health Systems Inc, North Tonawanda, NY, 1997.

25. Baron-Cohen S, Wheelwright S, Hill J, Raste Y, Plumb I. The "Reading the Mind in the Eyes" Test revised version: a study with normal adults, and adults with Asperger syndrome or highfunctioning autism. J Child Psychol Psychiatry 2001; 42: 241-251.

26. Wheelwright S, Auyeung B, Allison C, Baron-Cohen S. Defining the broader, medium and narrow autism phenotype among parents using the Autism Spectrum Quotient (AQ). Molec Autism 2010; 1: 1-10.

27. Bagby RM, Parker JDA, Taylor GJ. The twenty-item Toronto Alexithymia Scale-I. Item selection and cross-validation of the factor structure. J Psychosom Res 1994; 38: 23-32.

28. Bellinger DC, Wypij D, Rivkin MJ, et al. Adolescents with d-transposition of the great arteries corrected with the arterial switch procedure. Circulation 2011; 124: 1361-1369.

29. Newburger JW, Sleeper LA, Bellinger DC, et al. Early developmental outcome in children with hypoplastic left heart syndrome and related anomalies: the single ventricle reconstruction trial. Circulation 2012; 125: 2081-2091.

30. Marino BS, Lipkin PH, Newburger JW, et al. American Heart Association Congenital Heart Defects Committee, Council on Cardiovascular Disease in the Young, Council on Cardiovascular Nursing, and Stroke Council. Neurodevelopmental outcomes in children with congenital heart disease: evaluation and management: a scientific statement from the American Heart Association. Circulation 2012; 126: 1143-1172. 